\title{
D $+\mathrm{CH}_{4}$ 反应的 SVRT 含时波包理论研究 *
}

\author{
刘新国张庆刚 \\ (山东师范大学物理系, 济南 250014)
}

\begin{abstract}
摘要 基于 Jordan 和 Gilbert 势能面, 用 SVRT (semirigid vibrating rotor target)模型, 对 $\mathrm{D}+\mathrm{CH}_{4}$ 反应进行了含时 波包动力学研究, 计算得到了不同初始振动转动态的总反应几率、积分散射截面和热速率常数. 计算结果与 $\mathrm{H}+\mathrm{CH}_{4}$ 反应进行了比较和讨论. 反应几率随平动能的变化曲线呈现出显著的量子共振特性. 通过对 $j=0$ 时, $v=0 、 1 、 2$ 的反应几率的计算, 看出 $\mathrm{H}-\mathrm{CH}_{3}$ 的振动激发极大地提高了反应几率, 而反应阈能明显降低, 说明反应 分子的振动能对分子的碰撞反应有重要贡献. 对 $v=0$ 时, $j=0 、 1 、 2 、 3$ 的反应几率的计算, 得出转动量子数 $j$ 的增大也会使反应几率有较大的提高,但反应阈能基本不变.
\end{abstract}

关键词: 散射截面, 速率常数, 含时波包 中图分类号: 0643

近十多年来, 含时波包法以其在分子反应动力 学领域的优越性而成为研究反应散射非常有效的 手段. 用此法, 人们已经对大量的原子与双原子分 子及有限的几个四原子体系的反应进行了严格的 量子动力学研究. 然而, 由于化学和生物感兴趣的 绝大多数反应是四原子以上的多原子体系反应, 为 进行精确的量子计算, 发展一种适用于多原子反应 的实际可行的计算方法是非常必要的.为此人们 提出了很多约化维数的方法 ${ }^{[1-6]}$, 并且得到了许多有 用的结论 ${ }^{[4,7-8]}$. SVRT 模型 ${ }^{[9]}$ 已成功地用于一些多 原子分子碰撞反应研究 ${ }^{[10-11]}$, 本文将该模型应用于 一个典型的六原子体系反应, $\mathrm{D}+\mathrm{CH}_{4} \rightarrow \mathrm{CH}_{3}+\mathrm{HD}$, 并采用含时波包法 ${ }^{[12]}$ 对该反应进行量子动力学计 算, 完善和验证该模型对于多原子体系量子散射计 算的应用及为该体系的实验研究提供了理论依据.

\section{SVRT 模型应用于 $\mathrm{D}+\mathrm{CH}_{4} \rightarrow \mathrm{CH}_{3}+$ HD 反应}

\section{1 单原子 - 多原子分子反应的 SVRT 模型及 其哈密顿量}

在 SVRT 模型中, 靶分子被看作一个半刚性的 振转子, 它的空间运动可视为一个非对称转子的运 动, 包括一维相对运动 (振动) 和绕分子轴的转动. 在单原子 - 多原子反应的基本 SVRT 模型中, 人射
粒子 $\mathrm{A}$ (这里为 $\mathrm{D}$ 原子) 与 $\mathrm{SVRT}$ 分子 (这里为 $\mathrm{CH}_{4}$ ) 的哈密顿算符:

$$
\begin{aligned}
\hat{\mathbf{H}}_{\mathrm{ap}}= & -\frac{\hbar^{2}}{2 \mu} \frac{\partial^{2}}{\partial R^{2}}+\frac{\hat{\mathbf{L}}^{2}}{2 \mu R^{2}}+\hat{\mathbf{H}}_{\mathrm{T}}+\hat{\mathbf{V}} \\
= & -\frac{\hbar^{2}}{2 \mu} \frac{\partial^{2}}{\partial R^{2}}+\frac{\hat{\mathbf{L}}^{2}}{2 \mu R^{2}}+\frac{1}{2} \sum_{i j} \hat{\Pi}_{i} G_{i j} \hat{\Pi}_{j}- \\
& \frac{\hbar^{2}}{2 \mu_{\mathrm{T}}} \frac{\partial^{2}}{\partial r^{2}}+\hat{\mathbf{V}}_{\mathrm{T}}(r)+\hat{\mathbf{V}}
\end{aligned}
$$

这里: 下角 ap 表示的是原子 - 多原子分子 (atompolyatomic molecule), $\mu=\frac{M_{\mathrm{D}} M_{\mathrm{CH}_{i}}}{M_{\mathrm{D}}+M_{\mathrm{CH}_{4}}}$ 是反应体系的 约化质量, $\mu_{\mathrm{T}}=\frac{M_{\mathrm{H}} M_{\mathrm{CH}_{3}}}{M_{\mathrm{H}}+M_{\mathrm{CH}_{2}}}$ 为 $\mathrm{H}$ 和 $\mathrm{CH}_{3}$ 的约化质量, $R$ 是 $\mathrm{D}$ 原子和 $\mathrm{CH}_{4}$ 分子质心之间的距离, $r$ 为任一 $\mathrm{H}$ 原子与 $\mathrm{CH}_{3}$ 部分的质心之间的距离, $\hat{\mathbf{L}}$ 是轨道角 动量算符, $\hat{\Pi}_{i}$ 为转动角动量算符, $\hat{\mathbf{H}}_{\mathrm{T}}$ 为靶分子 $\mathrm{CH}_{4}$ 的哈密顿量算符. $\hat{\mathbf{V}}_{\mathrm{T}}(r)$ 表示 SVRT 分子(这里是 $\mathrm{CH}_{4}$ 分子) 的相互作用势算符, $\mathrm{T}$ 表示 (target molecule) 靶分子, 也就是 SVRT 分子 $\left(\mathrm{CH}_{4}\right.$ 分子 $) ; \hat{\mathbf{V}}$ 表示 D 原子与 $\mathrm{CH}_{4}$ 分子的相互作用势算符. D+ $\mathrm{CH}_{4}$ 反应体系的雅可比坐标如图 1 所示. 这里, 相 互作用势 $\hat{\mathbf{V}}$ 依赖于四个坐标: $R, r, \theta, \chi$. 其中极角 $\theta$ 为 $R$ 与 $r$ 间的夹角, $\chi$ 角为靶分子绕分子 $r$ 轴的 转角, $G$ 是转动惯量 $I$ 的倒数, 即 $G=1 / I$.

\section{2 含时波包传播}

2003-10-14 收到初稿, 2003-12-10 收到修改稿。联系人: 刘新国 (E-mail: liuxinguo@ sdnu. edu. cn; Tel: 0531-6180892; Fax: 0531-6180348)。*国家自然科学基金(19874040, 10174046)资助项目 
含时薛定谔方程:

$$
i \hbar \frac{\partial}{\partial t} \Psi(t)=\hat{\mathbf{H}} \Psi(t)
$$

将含时波函数按平动 - 振动 - 转动本征函数展开:

$$
\Psi(t)=\sum_{p, v, j, k, n} U_{p}^{v}(R) Z_{j k n}^{J M}(\Omega, \theta, \chi) \phi_{v}(r) C_{p j k n}(t)
$$

其中, $v$ 表示 $\mathrm{H}-\mathrm{CH}_{3}$ 的振动量子数, $j$ 和 $k$ 分别表 示转动角动量量子数和它在空间固定 (space-fixed) 轴 $R$ 轴上的投影, $n$ 表示 $j$ 在分子轴 $r$ 轴上的投影. $U_{p}^{v}$ 为平动基函数, $\phi_{v}(r)$ 为振动基函数, $Z$ 为体定 (body-fixed) 总角动量本征函数, $\Omega$ 为欧拉角, $J$ 为 角动量, $M$ 为磁量子数, $C_{p v j k n}(t)$ 表示与时间有关的 展开系数. 为了有效地节省计算量, 含时波包通过 分裂算符法 (split operator method) 传播:

$$
\Psi(t+\Delta)=\mathrm{e}^{-i \hat{\mathbf{H}}_{0} \Delta / 2} \mathrm{e}^{-i \hat{\mathbf{U}} \Delta} \mathrm{e}^{-i \hat{\mathbf{H}}_{0} \Delta / 2} \Psi(t)
$$

$\hat{\mathbf{H}}_{0}$ 为参考哈密顿算符, $\hat{\mathbf{U}}$ 为有效势算符, $\Delta$ 为时间 步长, 其中

$$
\begin{gathered}
\hat{\mathbf{H}}_{0}=-\frac{\hbar^{2}}{2 M} \nabla_{R}^{2}-\frac{\hbar^{2}}{2 \mu_{\mathrm{T}}} \frac{\partial^{2}}{\partial r^{2}}+\hat{\mathbf{V}}_{\mathrm{T}}(r) \\
\hat{\mathbf{U}}=\hat{\mathbf{H}}_{\mathrm{rot}}+\hat{\mathbf{V}}=\frac{\hat{\mathbf{L}}^{2}}{2 \mu R^{2}}+\frac{1}{2} \sum_{i j} \hat{\Pi}_{i} G_{i j} \hat{\Pi}_{j}+\hat{\mathbf{V}}
\end{gathered}
$$

靶分子的振转本征函数记为 $\psi_{v n}^{j k}\left(\Omega_{\mathrm{T}}, r\right)$, 其中, $r$ 为 振动坐标, $\Omega_{\mathrm{T}}$ 为三个欧拉角, $M$ 为参考物质量. 它 满足薛定谔方程:

$$
\begin{aligned}
& {\left[\frac{1}{2} \sum_{i j} \hat{\Pi}_{i} G_{i j} \hat{\Pi}_{j}-\frac{\hbar^{2}}{2 \mu_{\mathrm{T}}} \frac{\partial^{2}}{\partial r^{2}}+V_{\mathrm{T}}(r)\right] \psi_{v n}^{j k}\left(\Omega_{\mathrm{T}}, r\right)} \\
& \quad=E_{v j n} \psi_{v n}^{j k}\left(\Omega_{\mathrm{T}}, r\right)
\end{aligned}
$$

\section{2 结果与讨论}

采用 Jordan 和 Gilbert 的从头计算势能面 ${ }^{[13]}$, 运用 SVRT 模型, 选取该反应体系 12 个内坐标中的 4 个自由度, 对反应 $\mathrm{D}+\mathrm{CH}_{4} \rightarrow \mathrm{CH}_{3}+\mathrm{HD}$ 进行了量 子动力学计算. 如图 1 所示, 靶分子 $\mathrm{H}-\mathrm{CH}_{3}$ 被看 作一个半刚性振动转子, 由一个 $\mathrm{H}$ 原子和 $\mathrm{CH}_{3}$ 两部

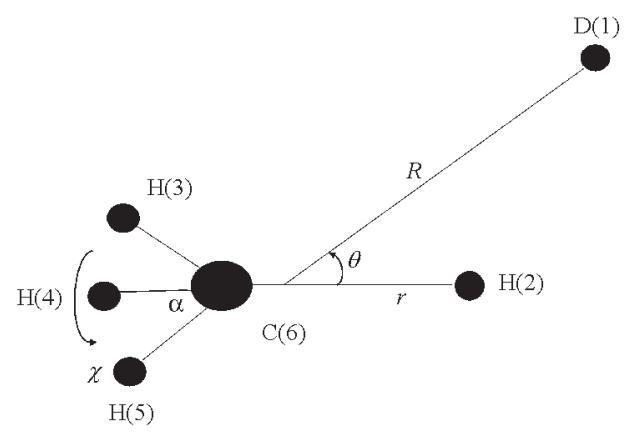

图 1 SVRT 模型应用于 $\mathrm{D}+\mathrm{CH}_{4}$ 反应

Fig. 1 SVRT model applied to reaction $\mathrm{D}+\mathrm{CH}_{4}$
分组成. 在反应过程中 $\mathrm{H}-\mathrm{CH}_{3}$ 保持 $C_{3}$ 对称, 所 有几何参数的选取都必须保持靶分子的 $C_{3}$ 对称. 比如 $\mathrm{CH}_{3}$ 部分任意两 $\mathrm{C}-\mathrm{H}$ 键间的夹角 $\alpha$ 在反应 前为 $109.47^{\circ}$, 过渡态时为 $107.45^{\circ}$, 反应后为 $90^{\circ}$, 这里选取其过渡态的值为 $107.45^{\circ}$, 当 $\alpha$ 取这个值 时, 能垒高度为 $46.1 \mathrm{~kJ} \cdot \mathrm{mol}^{-1}$, 与实验值 $46.1 \sim$ $50.2 \mathrm{~kJ} \cdot \mathrm{mol}^{-1}$ 最为接近. 计算中 $\mathrm{C}-\mathrm{H}$ 键的键长 也取其过渡态的值. 为得到充分收玫的结果, 在波 函数的展开式中共选取了 30 个振动基函数, 90 个 随 $R$ 变化的平动基函数 ( $R$ 的值从 $3.0 \mathrm{Bohr}$ 到 13. 5 Bohr), 分子的最大转动量子态达到 $j=35$, 初 始波包位于 $R=10.0 \mathrm{Bohr}$ 处. 在 SVRT 模型中 $\mathrm{CH}_{4}$ 的 4 个 $\mathrm{H}$ 原子被视为可区分的, 而在实际反应 中, $\mathrm{CH}_{4}$ 中的 4 个 $\mathrm{H}$ 原子是等同的, 不可区分的. 因此, 在最后的结果中, 实际的反应几率应乘以 4 .

\section{1 基态的反应几率}

图 2 给出了靶分子 $\mathrm{CH}_{4}$ 处于基态时的反应几 率. 由图 2 可以得出: (1)在平动能小于能垒高度 $0.477 \mathrm{eV}$ 时, 出现了反应几率不为 0 的现象, 在接 近能垒高度时, 反应几率已达到 0.066 , 说明量子隧 道效应非常显著. 这是因为反应中的约化质量很 小, 比一个 $\mathrm{D}$ 原子的质量还要小一点; (2)当平动能 继续增大时, 反应几率图像呈现出共振振荡结构, 这 是因为反应是在排斥势的作用下发生的, 且该反应 属于提取反应. 很多提取反应如 $\mathrm{H}+\mathrm{H}_{2}{ }^{[14]}, \mathrm{H}+$ $\mathrm{CH}_{4}{ }^{[11]}$ 等, 其反应几率随动能的变化都呈现出这种 类似共振的量子动力学振荡, 这种现象还有待进一 步的理论研究; (3)将基态时该体系的反应几率与 $\mathrm{H}+\mathrm{CH}_{4}$ 体系 ${ }^{[11}$ 进行比较可得, 基态时的反应阈能

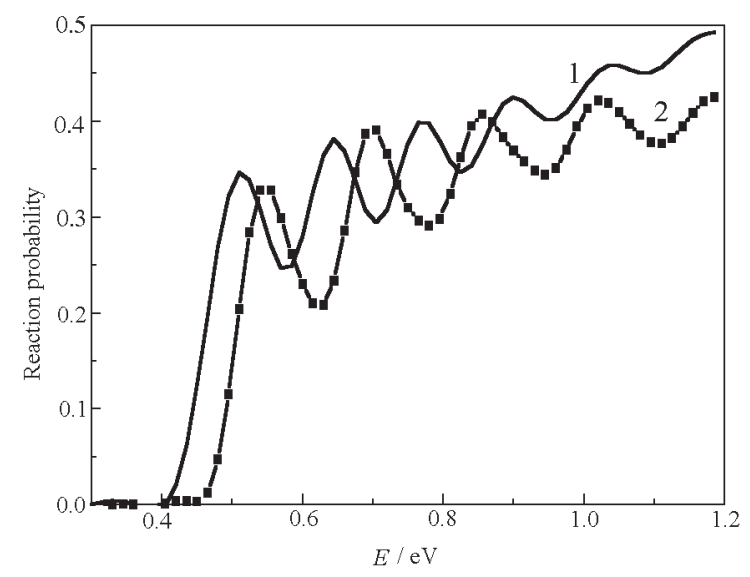

图 2 体系基态 $(v=j=0)$ 的反应几率

Fig. 2 Reaction probability of ground state $(v=j=0)$

1) $\left.\mathrm{D}+\mathrm{CH}_{4} ; 2\right) \mathrm{H}+\mathrm{CH}_{4}$ 


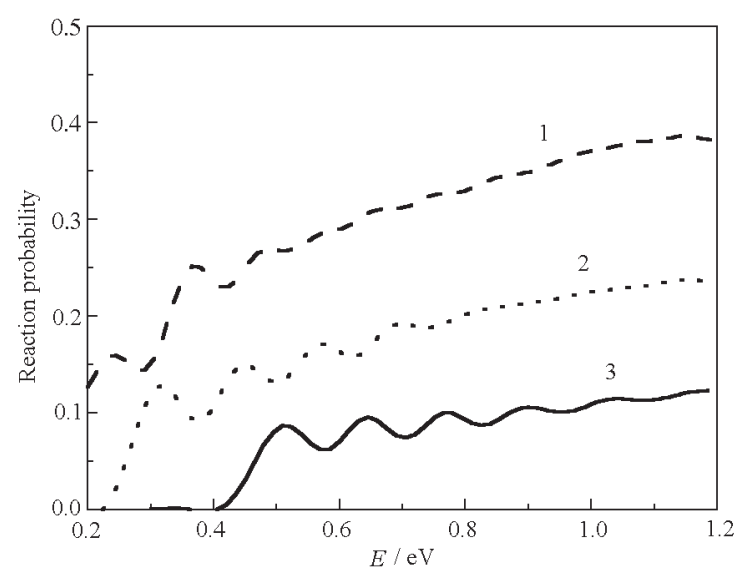

图 3 体系基态 $(v=0)$ 和振动激发态 $(v=1,2)$ 的反应几率

Fig. 3 Reaction probabilities of ground state

$(v=0)$ and vibrational excited $\operatorname{states}(v=1,2)$

1) $v=2,2) v=1,3) v=0 ; j=0$

比 $\mathrm{H}+\mathrm{CH}_{4}$ 体系要小, $\mathrm{D}+\mathrm{CH}_{4}$ 体系的反应國能为 $0.42 \mathrm{eV}$, 而 $\mathrm{H}+\mathrm{CH}_{4}$ 体系的反应阈能为 $0.46 \mathrm{eV}$, 这 是由同位素效应引起的. 因为 $\mathrm{D}+\mathrm{CH}_{4}$ 体系的平动 约化质量比 $\mathrm{H}+\mathrm{CH}_{4}$ 体系的平动约化质量大近一 倍, 从而使 $\mathrm{D}+\mathrm{CH}_{4}$ 体系的反应阈能比 $\mathrm{H}+\mathrm{CH}_{4}$ 体 系小了 $0.04 \mathrm{eV}$. 在计算的平动能范围内, $\mathrm{D}+\mathrm{CH}_{4}$ 体系的平均反应几率都高于 $\mathrm{H}+\mathrm{CH}_{4}$ 体系的反应几 率也是由于同样的原因.

\section{2 振动对反应的影响}

我们计算了不同初始振动激发态下 $\mathrm{D}+\mathrm{CH}_{4}$ 反 应的反应几率. 图 3 给出了 $j=0$ 时, 初始振动态 $v=0 、 1 、 2$ 时反应几率随平动能的变化曲线图. 可 以看出, 随着振动量子数的增大, 反应几率显著提 高, 且反应阈能明显降低. 当振动量子数由 $v=0$ 增至 $v=1$ 时, 反应阈能降低约 $0.19 \mathrm{eV}$, 随着振动 量子数 $v$ 的增大, 反应阈能继续降低, 但降低幅度 在变小, 说明反应分子的振动能对 $\mathrm{C}-\mathrm{H}$ 键的断裂 有很大的贡献.

\section{3 转动对反应的影响}

本文还计算了对应于振动基态下不同初始转动 激发态 $\mathrm{D}+\mathrm{CH}_{4}$ 反应的反应几率. 图 4 给出了 $v=$ 0 时, 初始转动态 $j=0 、 1 、 2 、 3$ 时的反应几率随平动 能的变化关系曲线. 由图 4 可知, 当 $j$ 由 0 变为 1 时, 反应几率有很大的提高, 增加了近两倍. 当 $j$ 由 1 变为 2 及由 2 增加到 3 时, 反应几率有更大的提 高, 但增加的幅度在减小. 另外, 反应几率随转动量 子数的增加是在相对比较低的碰撞能的条件下发生 的. 由图 4 可见, 对于初始转动态 $j=3$, 在相对高 的碰撞能时, 反应几率实际上在降低. 对于不同的

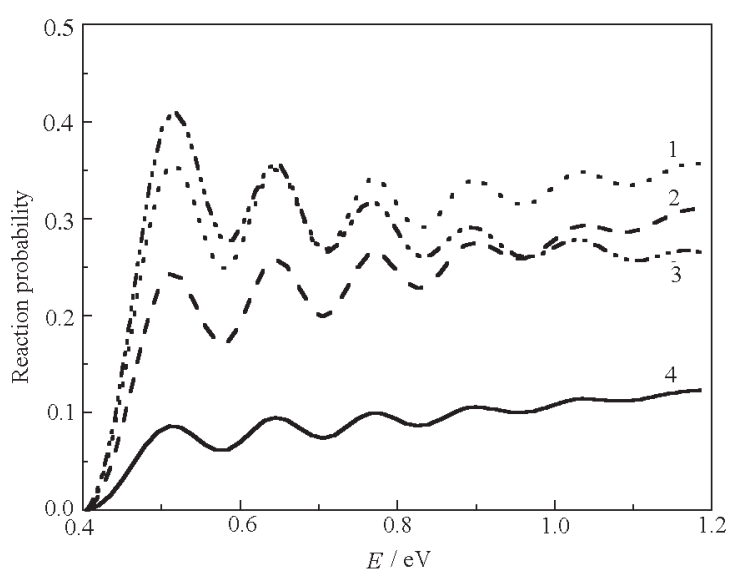

图 4 体系的不同转动态的反应几率

Fig. 4 Reaction probabilities from different rotational states

1) $j=2,2) j=1,3) j=3,4) j=0 ; v=0 ; k=n=0$

转动态, 虽然反应几率随平动能的变化规律不同, 但 反应國能基本不变, 说明反应分子的转动能的增加, 对提取反应有重要贡献, 但基本不影响反应阈能值.

\section{4 体系总的散射截面和速率常数}

为计算体系总散射截面和速率常数, 对于总角 动量 $J>0$ 的情况, 采用 C-S (centrifugal sudden) 近 似. 为保证取得充分收玫的结果, 计算中体系的总 的角动量 $J$ 取到最大值 $J=64$. 体系的总的散射截 面 $\sigma$ 和速率常数 $(k)$ 的计算公式如下:

$$
\begin{gathered}
\sigma_{v}(E)=\frac{\pi}{k_{\mathrm{B}}^{2}} \sum_{J}(2 J+1) \mathrm{P}_{v}^{J}(E) \\
k(T)=\left(\frac{8 k_{\mathrm{B}} T}{\pi \mu}\right)^{\frac{1}{2}}\left(k_{\mathrm{B}} T\right)^{-2} \int_{0}^{\infty} \mathrm{d} E_{t} \cdot \\
E_{t} \exp \left(-E_{t} / k_{\mathrm{B}} T\right) \sigma_{v}\left(E_{t}\right)
\end{gathered}
$$

式中 $k_{\mathrm{B}}$ 为玻尔兹曼常数. 图 5 给出了对应于反应 物 $\mathrm{CH}_{4}$ 的基态和第一激发态的总的散射截面随平 动能变化的曲线. 由图 5 可以看出, $\mathrm{D}+\mathrm{CH}_{4}$ 体系 的总散射截面随人射平动能的增大而增大. 与基态 的 $\mathrm{H}+\mathrm{CH}_{4}$ 体系 ${ }^{[11}$ 的总散射截面结果比较, $\mathrm{D}+\mathrm{CH}_{4}$ 体系基态的散射截面在计算的整个平动能范围内都 比 $\mathrm{H}+\mathrm{CH}_{4}$ 体系的结果大, 这也与前面 $\mathrm{D}+\mathrm{CH}_{4}$ 体 系的反应几率的增大是一致的. 此外, 由图 5 还可 看出, $\mathrm{D}+\mathrm{CH}_{4}$ 体系的振动激发态的散射截面远比 基态的散射截面大, 这说明振动激发极大地增加了 反应的散射截面. 图 6 给出了对应于反应物 $\mathrm{CH}_{4}$ 的基态和第一激发态的速率常数曲线. 由图 6 可以 看出, 与基态的 $\mathrm{H}+\mathrm{CH}_{4}$ 体系 ${ }^{[11}$ 的速率常数结果比 较, $\mathrm{D}+\mathrm{CH}_{4}$ 体系的基态的速率常数在 $200 \sim 1000$ $\mathrm{K}$ 的温度范围内比 $\mathrm{H}+\mathrm{CH}_{4}$ 体系的结果大, 而在 1 


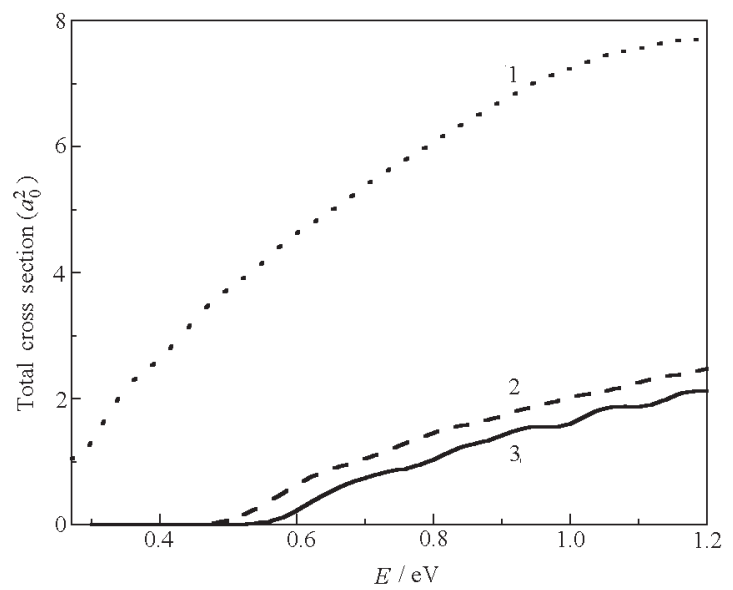

图 5 体系基态 $(v=0)$ 和振动激发态 $(v=1)$ 总散射截面

Fig. 5 Total cross-section of ground state $(v=0)$ and vibrational excited state $(\boldsymbol{v}=\mathbf{1})$

1) $\left.\left.\mathrm{D}+\mathrm{CH}_{4}, v=1 ; 2\right) \mathrm{D}+\mathrm{CH}_{4}, v=0 ; 3\right) \mathrm{H}+\mathrm{CH}_{4}, v=0$;

$a_{0}$ : Bohr radius

$000 \sim 2000 \mathrm{~K}$ 的温度范围内, 二者的结果几乎是相 同的. 此外, $\mathrm{D}+\mathrm{CH}_{4}$ 体系的振动激发态的速率常 数远大于基态的速率常数, 说明振动激发极大地增 加了反应的速率常数, 振动激发更有利于反应的进 行.

\footnotetext{
References

Sun, Q. ; Bowman, J. M. J. Chem. Phys., 1990, 92: 5201

Brook, A. N. ; Clary, D. C. J. Chem. Phys., 1990, 92: 4178

Takayanagi, T. J. Chem. Phys., 1996, 104: 2237
}

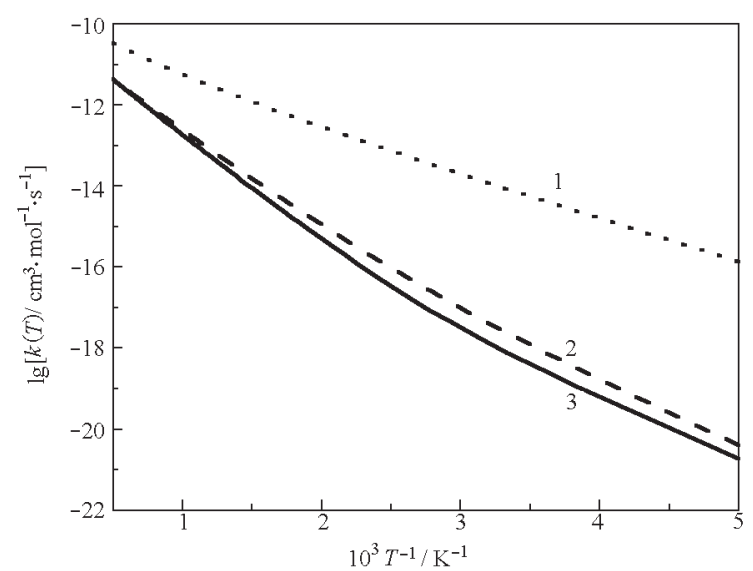

图 6 体系基态 $(v=0)$ 和振动激发态 $(v=1)$ 的速率常数

Fig. 6 Rate constants of ground state $(v=0)$ and vibrational excited state $(v=1)$

1) $\left.\left.\mathrm{D}+\mathrm{CH}_{4}, v=1 ; 2\right) \mathrm{D}+\mathrm{CH}_{4}, v=0 ; 3\right) \mathrm{H}+\mathrm{CH}_{4}, v=0$

\footnotetext{
Yu, H. G. ; Nyman, G. J. Chem. Phys., 1999, 111: 3508

Larranaga, F. H. ; Manthe, U. J. Chem. Phys., 2000, 113: 5115

Wang, D. Y. ; Bowman, J. M. J. Chem. Phys., 2001, 115: 2055

Bowman, J. M. ; Wang, D. J. Chem. Phys., 1992, 96: 7852

Balakrishnan, N. ; Billing, G. D. J. Chem. Phys., 1994, 101: 2785

Zhang, J. Z. H. J. Chem. Phys., 1999, 111: 3929

Zhang, D. H. ; Zhang, J. Z. H. J. Chem. Phys., 2000, 112: 585

11 Wang, M. L. ; Li, Y. M. ; Zhang, J. Z. H. J. Chem. Phys., 2000, 113: 1802

12 Zhang, D. H. ; Zhang, J. Z. H. J. Chem. Phys., 1994, 101: 1146

13 Jordan, M. J. T. ; Gilbert, R. G. J. Chem. Phys., 1995, 102: 5669

14 Miller, W. H. Annu. Rev. Phys. Chem., 1990, 41: 245
}

\title{
Time-dependent Wave Packet Theoretical Study for D $+\mathrm{CH}_{4}$ Reaction with SVRT Model *
}

Liu Xin-Guo Zhang Qing-Gang

( Department of Physics, Shandong Normal University, Jinan 250014)

\begin{abstract}
The SVRT (semirigid vibrating rotor target) model is applied to study the reaction of $\mathrm{D}+\mathrm{CH}_{4} \mathrm{using}$ time-dependent wave packet method based on the PES of Jordan and Gilbert. The total reaction probabilities, cross-sections and rate constants of different initial rovibrational states are calculated. The comparison and discussion are carried out between the calculated results for $\mathrm{D}+\mathrm{CH}_{4}$ and that for $\mathrm{H}+\mathrm{CH}_{4}$. The energy dependence of the calculated reaction probability shows oscillatory structures, similar to those observed in the reactions $\mathrm{H}+\mathrm{H}_{2}, \mathrm{H}+$ $\mathrm{CH}_{4}$ etc. We have also studied the influence of internal energy of the reactant molecule $\left(\mathrm{CH}_{4}\right)$ on the reaction probability. The excitation of the $\mathrm{H}-\mathrm{CH}_{3}$ stretching vibration gives significant enhancement of reaction probability and the reaction threshold decreases with the enhancement of the vibrational excitation. And the reaction probability also rises significantly with the enhancement of rotational quantum number $j$ but the reaction threshold has no change.
\end{abstract}

Keywords: Scattering cross-section, Rate constant, Time-dependent wave packet

Received: October 14, 2003; Revised: December 10, 2003. Correspondent: Liu Xin-Guo(E-mail: liuxinguo @ sdnu. edu. cn; Tel: 0531-6180892; Fax: 0531-6180348). $\quad{ }^{*}$ The Project Supported by NSFC (19874040, 10174046) 